\title{
The Sun in Time: Evolution of Coronae of Solar-Type Stars
}

\author{
MANUEL GÜDEL ${ }^{1}$ AND EDWARD F. GUINAN ${ }^{2}$
}

${ }^{1}$ Paul Scherrer Institut, CH-5232 Villigen PSI, Switzerland, and JILA, University of Colorado, Boulder, CO 80309-0440

${ }^{2}$ Department of Astronomy and Astrophysics, Villanova University, Villanova, PA 19085

We report on the results of a multi-frequency program to study coronal X-ray, EUV, and microwave activity of solar-type $(\mathrm{G} 0-5 \mathrm{~V})$ stars of greatly different ages. These stars are of interest as proxies for the Sun for ages from $50 \mathrm{Myr}$ to $10 \mathrm{Gyr}$. Coronal temperatures decrease with X-ray/EUV luminosity and with increasing age and rotation period according to power-law relations. The young Sun had an extremely luminous corona with $L_{\mathrm{EUV}, \mathrm{X}}=$ $100-600 L_{\mathrm{EUV}} \mathrm{x}$ (present Sun) and temperatures up to $10 \mathrm{MK}$; this information is pivotal for the study of the young planetary atmospheres. The findings further suggest an intimate connection between the presence of very hot coronal plasma and non-thermal radio-emitting particles.

\section{A Diary of Solar Life: An Age Sequence of Solar Proxies}

The Sun's magnetic activity and consequently its chromospheric and coronal emissions are expected to have declined steadily to present levels as the solar rotation slowed due to magnetic braking (Skumanich 1972). Despite the fact that evolutionary models indicate that the Sun was less luminous in its early ZAMS phase $\left(L_{\mathrm{ZAMS}} \approx 0.76 L_{\odot}\right)$, Canuto et al. (1982) have estimated that in the Sun's pre-main-sequence T Tauri phase, solar X-ray, EUV, and UV emissions were enhanced by up to hundreds of times present levels. Direct observation of high-energy emissions from younger solar-type stars is the only means of establishing the level of the solar short wavelength flux at earlier epochs.

Table 1 presents our sample of solar proxies (spectral types G0-G5 V), selected mainly for their well established rotation periods (as chiefly measured by optical photometry) and reliably determined ages (partly supported by cluster or moving group memberships, agerotation relations, and isochrone ages). The ages of the targets range from approximately $70 \mathrm{Myr}$ (near-zero-age main-sequence = ZAMS; EK Dra) to $8.8 \mathrm{Gyr}$ (terminal-age mainsequence $=$ TAMS $; \beta$ Hydri). For most of these stars, we obtained ROSAT PSPC observations in the Guest Observer Program; further, ASCA and EUVE data are being collected, and a vast amount of VLA, IUE and optical data obtained over the last decade clearly makes this an exciting moment to assemble bits and pieces of information into a more coherent picture on the long-term evolution of solar magnetic activity.

\section{Dissecting the Sun's Activity in Time: Previous Findings}

Coronal parameters are expected to vary with the efficiency of the magnetic dynamo. For example, Güdel et al. (1995a) found rotational modulation in the cooler X-ray component of the young EK Dra; through geometric modeling, they determined that

- the cool plasma of $\sim 2 \mathrm{MK}$ is confined to heights (above the photosphere) similar to active regions in the quiet solar corona, i.e., $\sim 0.1 R_{\odot}$. Its coronal filling factor is moderate, showing that young solar proxies possess well localized, magnetically confined structures that we identify with "nonflaring stellar active regions".

- The electron density of the cool component exceeds $4 \cdot 10^{10} \mathrm{~cm}^{-3}$. 
TABLE 1. Coronal properties of the Sun in time (X-ray data from present observations, or Güdel et al. 1995b or Dorren \& Guinan 1994; stellar $8.5 \mathrm{GHz}$ radio data from Güdel et al. 1994, $1995 \mathrm{ab} ; L_{\mathrm{X}}$ for $\beta$ Hyi scaled to one solar radius)

\begin{tabular}{|c|c|c|c|c|c|c|c|}
\hline Star & $\begin{array}{l}\text { Spectr. } \\
\text { Type }\end{array}$ & $\begin{array}{l}P_{\text {rot }} \\
\text { (d) }\end{array}$ & $\begin{array}{l}\text { Age } \\
\text { (Gyr) }\end{array}$ & $\begin{array}{l}\log L_{\mathrm{X}} \\
\left(\mathrm{erg} \mathrm{s}^{-1}\right)\end{array}$ & $\begin{array}{l}T_{\text {hot }} / T_{\text {cool }} \\
(\mathrm{MK})\end{array}$ & $\begin{array}{l}\mathrm{EM}_{\text {hot }} / \\
\mathrm{EM}_{\text {cool }}\end{array}$ & $\begin{array}{l}\log L_{\mathrm{R}} \\
\left(\mathrm{erg} \mathrm{s}^{-1} \mathrm{~Hz}^{-1}\right)\end{array}$ \\
\hline EK Dra & Go V & 2.75 & 0.07 & 29.92 & $9.33_{-.78}^{+.67} / 2.29_{-.29}^{+.34}$ & $\geq 1.16$ & $14.1 \pm 0.5$ \\
\hline$\pi^{1} \mathrm{UMa}$ & $\mathrm{G} 1.5 \mathrm{~V}$ & 4.68 & 0.3 & 29.09 & $6.35_{-71}^{+.71} / 1.41_{-.12}^{+.14}$ & $\overline{1.15}$ & $\leq 13.37$ \\
\hline HN Peg & Go V & 4.86 & 0.3 & 28.95 & $\ldots$ & $\ldots$ & $\overline{\leq} 13.38$ \\
\hline$\chi^{1}$ Ori & G1 V & 5.08 & 0.3 & 29.13 & $7.65_{-.55}^{+.86} / 1.56_{-.18}^{+.27}$ & 0.65 & $\ldots$ \\
\hline HD 1835 & $\mathrm{G} 2 \mathrm{~V}$ & 7.65 & 0.6 & 29.13 & $6.52_{-1.2}^{+.98} / 1.62_{-.37}^{+.28}$ & 0.79 & $\ldots$ \\
\hline VB 64 & G2 V & 8.7 & 0.6 & 29.41 & $\ldots$ & $\ldots$ & $\ldots$ \\
\hline$\kappa^{1}$ Cet & G5 V & 9.2 & 0.7 & 28.82 & $7.21_{-1.9}^{+1.6} / 1.74_{-.25}^{+.20}$ & 0.45 & $\ldots$ \\
\hline$\beta$ Com & Go V & 12.4 & 1.6 & 28.11 & $3.66_{-57}^{+1.6} / 1.24_{-.11}^{+11}$ & 0.45 & $\ldots$ \\
\hline 15 Sge & G5 V & 13.5 & 1.9 & 28.05 & $3.68_{-2.0}^{+1.9} / 1.06_{-36}^{+.38}$ & 0.60 & $\ldots$ \\
\hline Sun & $\mathrm{G} 2 \mathrm{~V}$ & 25.4 & 4.6 & 27.3 & $3.03_{-.32}^{+.56} / 1.22_{-.11}^{+}$ & 0.74 & 10.7 \\
\hline$\alpha$ Cen A & G2 V & $\sim 30$ & $5-6$ & 27.11 & $\ldots$ & $\ldots$ & $\ldots$ \\
\hline$\beta \mathrm{Hyi}$ & G2 IV & $\sim 45$ & 9 & 27.08 & $2.09_{-.18}^{+.31}$ & $\ldots$ & $\ldots$ \\
\hline
\end{tabular}

- Thus, a significant portion of the high (non-flaring) X-ray luminosity $L_{\mathrm{X}}$ in EK Dra is not due to large volumes but rather due to high plasma density. This in turn requires, for plasma confinement, stronger average coronal magnetic fields than on the Sun.

EK Dra is the first solar proxy discovered as a non-thermal radio source (Güdel, Schmitt, \& Benz 1994), evident both in quiescent and flaring emission (Güdel et al. $1995 \mathrm{a}$ ). This shows that the young Sun was a source of copious acceleration of nonthermal particles, producing a radio luminosity $L_{\mathbf{R}}$ more than 3 orders of magnitude higher than the quiet Sun's (which is thermal emission from lower atmospheric levels).

Slightly older solar-type stars continue to keep their strong non-thermal radio emission for some time. The rapidly rotating G5V star Gliese 755, estimated to be approximately $200 \mathrm{Myr}$ old, was detected at $\log L_{\mathrm{R}}=14.03\left(\log L_{\mathrm{X}}=29.52\right)$, while, on the other hand, HN Peg and $\pi^{1}$ UMa remained undetected ( $\left.\log L_{\mathrm{R}} \leq 13.37\right)$, indicating a time of the order of $300 \mathrm{Myr}$ for the decay of steady radio emission to below $\log L_{\mathrm{R}}=13.5$.

\section{Coronal Cooling with Age}

The coronal $L_{\mathbf{X}}$ of a GV star is well correlated with its rotation period $P_{\text {rot }}$ and consequently with its age, with $L_{\mathrm{X}} \propto P_{\text {rot }}^{-2}$ (Dorren, Guinan, \& DeWarf 1994).

In Fig. 1a, an age sequence of ROSAT spectra of $\mathrm{G}$ stars is shown, with each ROSAT spectrum renormalized to a constant amplitude of the lower-energy peak. The quiet solar corona was modeled using the continuous emission measure (EM) distribution given in Raymond \& Doyle (1981) between 1 and 5 MK (supplemented with realistic noise). All spectra were fitted with two Raymond-Smith type thermal plasma components. Notice that the Sun's $L_{\mathrm{X}}$ is close to $\beta$ Hyi's, despite the latter's significantly higher age $(8.8 \mathrm{Gyr}$; see Dorren, Güdel, \& Guinan 1995). This is because $\beta$ Hydri has, as a consequence of its leaving the main sequence, expanded to a radius of $1.6 R_{\odot}$. Its surface X-ray flux is, however, $\sim 2 \frac{1}{2}$ times smaller than the Sun's.

In our stars, the low-energy peaks contain photons from both plasma components (typically with comparable contributions), while the bump around $0.8 \mathrm{keV}$ is largely 

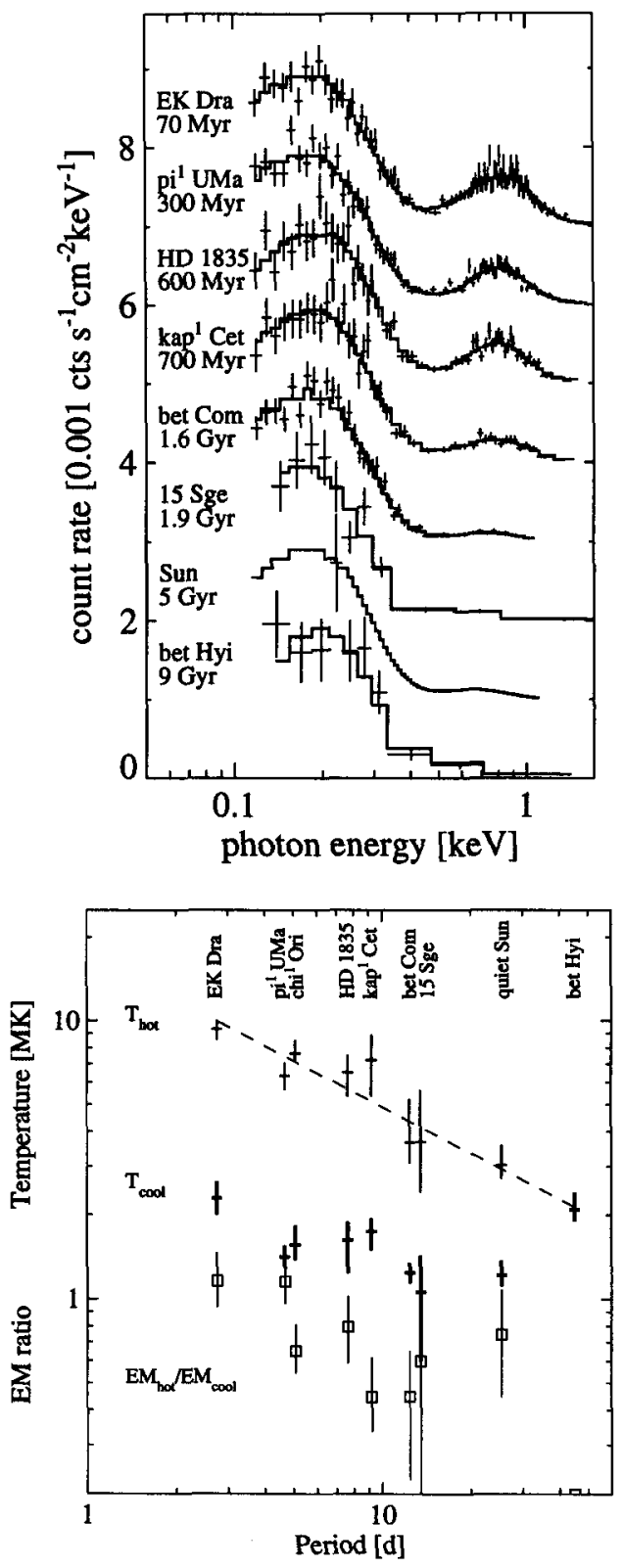

FIGURE 1. a (top): ROSAT PSPC pulse-height spectra of the solar proxy age series, normalized such that the left (softer) peak is about equal for all spectra. Individual spectra have been shifted by multiples of $0.001 \mathrm{cts} \mathrm{s}^{-1} \mathrm{~cm}^{-2} \mathrm{keV}^{-1}$ for illustration. Age monotonically increases from top (EK Dra: $70 \mathrm{Myr}$ ) to bottom ( $\beta$ Hydri: $8.8 \mathrm{Gyr}$ ). The consistent decrease of hard emission in the peak around $0.8 \mathrm{keV}$ indicates a decrease in EM at high $T$. Crosses are observations, while histograms represent fit (2-T Raymond-Smith). b (bottom): Coronal $T_{\mathrm{X}}$ and EM ratios for the same stars, plotted versus $P_{\text {rot }}$. Thin: Hotter PSPC temperature; dashed line represents a power-law. Bold: Cooler PSPC temperature. Quadrangles: EM ratio of hot and cool temperature. 
determined by hot (5-15 MK) plasma. Quite evidently, the relative amount of hot plasma and therefore hard radiation diminishes with age.

Fig. $1 \mathrm{~b}$ shows the numerical fit results. The higher temperature decreases from around $10 \mathrm{MK}$ near the ZAMS age $\left(P_{\text {rot }} \approx 2 \mathrm{~d}\right)$ to $\sim 2-3 \mathrm{MK}$ beyond the age of the Sun. For $T_{\text {hot }}$, we thus find a decay law

$$
\begin{gathered}
T_{\text {hot }}=1.74 \cdot 10^{7} P_{\mathrm{rot}}^{-0.55 \pm 0.03}[\mathrm{~K}], \\
T_{\text {hot }}=4.7 \cdot 10^{6} t^{-0.31}[\mathrm{~K}],
\end{gathered}
$$

where $P_{\text {rot }}$ is in days and $t$, the stellar age, is in Gyr. The second equation has been derived using a rotation-age relation for $\mathrm{G}$ stars, $\log t=1.75 \log P_{\mathrm{rot}}-1.81$ ( $t$ in Gyr, $P_{\text {rot }}$ in d; Dorren et al. 1994).

The nearly consistent decrease of the $\mathbf{E M}$ ratios for the hotter stars (i.e., $\mathbf{E M}_{\text {hot }} / \mathbf{E M}_{\text {cool }}$; quadrangles in Fig. 1b) further suggests that the hotter plasma not only becomes cooler with age, but also that it becomes less abundant, and thus that the distribution of plasma falls off increasingly steeper with age (starting from nearly flat in young stars). Further, the variation of $T_{\text {cool }}$ along with $T_{\text {hot }}$ is indicative of a continuous $E M$ distribution: apparently, the whole distribution shifts toward cooler temperatures with increasing age, although the ROSAT PSPC spectral fits are not sensitive enough to reveal more than two plasma components. From Fig. $2 \mathrm{a}, T_{\text {hot }}$ is correlated with the total $L_{\mathrm{X}}$ :

$$
L_{\mathrm{X}} \approx 84 T_{\text {hot }}^{3.97 \pm 0.23} \approx 50 T_{\text {hot }}^{4}\left[\mathrm{erg} \mathrm{s}^{-1}\right]
$$

( $T$ in $\mathrm{K}$ ). Remarkably, this remains true if we add close binary systems consisting of two almost identical solar-type G stars each: ER Vul (G1V+G1V), HR 8358 (G5V+G5V), and TZ CrB (G0V+G0V). The data for these binaries are from Dempsey et al. (1993). To account for binarity, their $L_{\mathbf{X}}$ were divided by two. Similar relations have been reported for less restricted star samples (e.g., Schrijver, Mewe, \& Walter 1984). For a theoretical discussion of such relations, see Jordan \& Montesinos (1991).

Since $L_{\mathrm{R}} \propto L_{\mathrm{X}}$ in active G stars (Güdel et al. 1995b), we find $L_{\mathrm{R}} \approx 1.6 \cdot 10^{-14} T_{\text {hot }}^{4}$. It implies that microwave luminous stars are those with hot coronae, and particle acceleration is intimately connected to the production of very hot (thermal) plasma.

\section{EUV Properties: Their Relevance to Planetary Atmospheres}

We summarize EUV properties of solar analogs as obtained from ROSAT/Wide Field Camera Survey observations (Fig. 2b). The EUV luminosity roughly fulfills a relation similar to the $\mathrm{X}$-rays (we give both relations here),

$$
\begin{gathered}
L_{\mathrm{EUV}} \approx 5.3 \cdot 10^{29} P_{\mathrm{rot}}^{-2.06 \pm 0.15} \approx 3.8 \cdot 10^{27} t^{-1.17}\left[\mathrm{erg} \mathrm{s}^{-1}\right] \\
L_{\mathrm{X}} \approx 8.4 \cdot 10^{30} P_{\mathrm{rot}}^{-2.43 \pm 0.15} \approx 2.6 \cdot 10^{28} \mathrm{t}^{-1.39}\left[\mathrm{erg} \mathrm{s}^{-1}\right]
\end{gathered}
$$

( $P_{\text {rot }}$ in days, $t$ in Gyr). The much increased EUV and $\mathrm{X}$-ray emissions of the "younger Suns" are of primary relevance for the formation of planetary ionospheres. In particular, they yield information on the young Sun's influence on the upper terrestrial atmosphere. Similarly, the enhanced UV emission is crucial for our understanding of the photochemistry in the young Earth's atmosphere.

\section{Summary and Conclusions}

The Sun's evolution from ZAMS to TAMS involves spin-down due to angular momentum loss in a magnetized wind. The feedback to the magnetic activity itself via an 

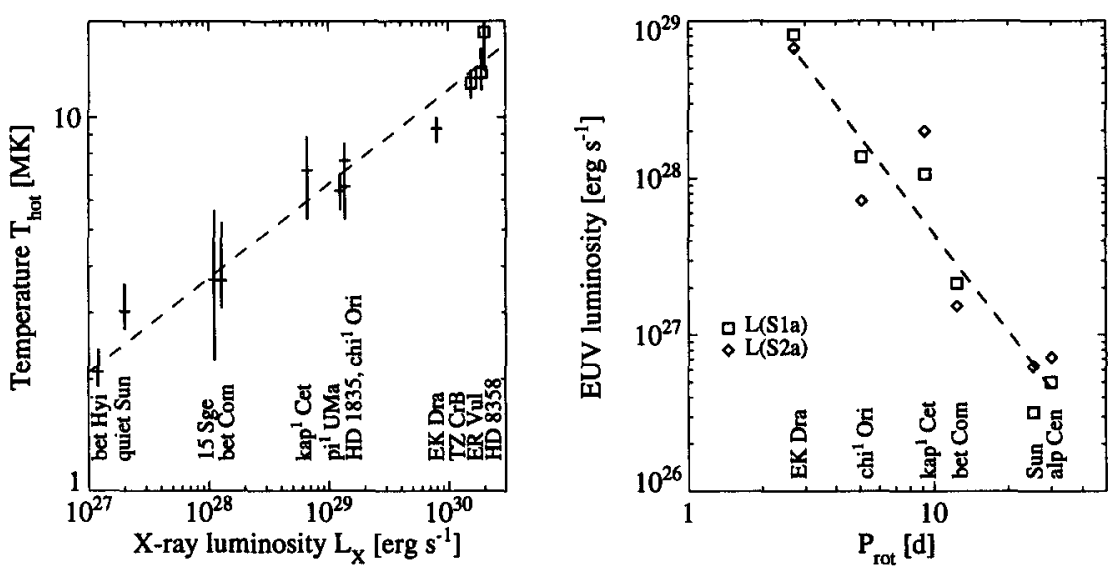

Figure 2. a (left): The coronal $T_{\text {hot }}$ versus $L_{\mathrm{X}}$ in the $[0.1,2.4] \mathrm{keV}$ energy range, clearly showing a strong correlation. The three stars indicated with quadrangles are three close binary systems consisting of two identical G dwarfs each (see text). b (right): ROSAT/WFC (Survey) luminosities for a sample of solar-like $G$ stars. Quadrangles and diamonds indicate luminosities in the S1 and in the S2 filters, respectively. The diagonal is a power-law (Eq. 4.4).

internal dynamo is theoretically poorly understood, but can be readily studied with the currently growing data pool of X-ray, EUV, UV, optical, and radio data of solar proxies at different ages. We find that the X-ray/EUV luminosity of solar-like stellar coronae decreases with the rotation period as $\sim P^{-2 \ldots-2.4}$, an that they become cooler with increasing $P_{\text {rot }}$ and thus age, with $T_{\text {hot }}=1.74 \cdot 10^{7} P^{-0.55}[\mathrm{~K}]$ and $T_{\text {hot }}=4.7 \cdot 10^{6} t^{-0.31}[\mathrm{~K}]$. By implication, $L_{\mathrm{X}} \approx 50 T_{\text {hot }}^{4}\left[\mathrm{erg} \mathrm{s}^{-1}\right.$ ].

Very hot quiescent plasma $\left(Z 10^{7} \mathrm{~K}\right)$ is restricted to young solar proxies. The same appears to be true for the persistent presence of a large amount of non-thermal radio emitting electrons detectable in microwaves. We thus find a three-fold tight correlation between $L_{\mathrm{R}}, L_{\mathbf{X}}$, and $T_{\text {hot }}$, indicating that particle acceleration is intimately related with the production of very hot coronae.

The ROSAT project has been supported by the Bundesministerium für Forschung und Technologie (BMFT) and the Max-Planck-Gesellschaft (MPG). This research has been supported by NASA ROSAT grants NAG5-1662 and NAG5-1703, and by NASA IUE grants NAG5-382 and NAG5-1703. We also acknowledge the support of NSF grant AST 86-16362. MG has been supported by the Swiss National Science Foundation (grant 8220-033360) and by NASA grant NAG5-1887 to the University of Colorado.

\section{REFERENCES}

Canuto, V. M., Levine, J. S., Augustsson, T. R. \& Imhoff, C. L. 1982, UV radiation from the young Sun and oxygen and ozone levels in the prebiological paleoatmosphere, Nature, 296, 816

Dempsey, R. C., Linsky, J. L., Schmitt, J. H. M. M. \& Fleming, T. A. 1993, The ROSAT All-Sky Survey of active binary coronae II: Coronal temperatures of the RS Canum Venaticorum Systems, ApJ, 413, 333 
Dorren, J. D. \& Gunan, E. F. 1994, The Sun in Time: Detecting and Modelling Magnetic Inhomogeneities on Solar-type Stars, in The Sun as a Variable Star, IAU Colloq. 143, ed. J. M. Pap et al., Cambridge, 206

Dorren, J. D., Guinan, E. F. \& DeWarf, L. E. 1994, The Decline of Solar Magnetic Activity with Age, in The 8th Cambridge Workshop on Cool Stars, Stellar Systems, and the Sun, ed. J.-P. Caillault, ASP Conf. Ser., 64, 399

Dorren, J. D., GüDEL, M., \& Guinan, E. F. 1995, X-Ray Emission from the Sun in its Youth and Old Age, ApJ, in press

GÜDEL, M., SchmitT, J. H. M. M. \& BeNZ, A. O. 1994, Discovery of microwave emission from four nearby solar-type G stars, Science, 265, 933

GüDel, M., SchmitT, J. H. M. M., Benz, A. O. \& Elias, N. M. II 1995a, The corona of the young solar analog EK Draconis, A\&A, in press

GüDEL, M., SchmitT, J. H. M. M. \& BenZ, A. O. 1995b, Microwave emission from X-ray bright solar-like stars: The F-G main-sequence and beyond, $A \& A$, in press

JoRDAN, C. \& MontEsinos, B. 1991, The dependence of coronal temperature son Rossby numbers, MNRAS, 252, 21 P

RAYMOND, J. C. \& DoYLE, J. G. 1981, Emissivities of strong ultraviolet lines, ApJ, 245, 1141

Schrijver, C. J., Mewe, R. \& Walter, F. M. 1984, Coronal activity in F-, G-, and K-type stars II: Coronal structure and rotation, A\&A, 138, 258

Skumanich, A. 1972, Time-scales for Ca II emission decay, rotational braking, and lithium depletion, ApJ, 171, 565 